\title{
300.
}

\section{NOTE RELATIVE AUX DROITES EN INVOLUTION DE M. SYLVESTER.}

[From the Comptes Rendus de l'Académie des Sciences de Paris, tom. LII. (JanvierJuin, 1861), pp. 1039-1042.]

LA courbe cubique dans l'espace, représentée par les équations

$$
y u-z^{2}=0, \quad z y-x u=0, \quad x z-y^{2}=0,
$$

passe par le point $A(x=y=z=0)$ et le point $B(y=z=u=0)$; le plan $x=0$ est le plan osculant en $A$, le plan $y=0$ le plan par la tangente en $A$ et la droite $A B$; le plan $z=0$ celui par la droite $A B$ et la tangente en $B$; et enfin le plan $u=0$ est le plan osculant en $B$. Réciproquement, pour une courbe cubique quelconque, en prenant les points $A, B$, sur la courbe à volonté, et en fixant comme ci-dessus les significations des coordonnées $x, y, z, u$, les facteurs constants que contiennent implicitement ces valeurs étant convenablement déterminés, les équations de la courbe cubique seront

$$
y u-z^{2}=0, \quad z y-x u=0, \quad x z-y^{2}=0 .
$$

Par un point quelconque de l'espace il passe une droite qui coupe deux fois la courbe cubique; et en prenant $\left(x_{1}, y_{1}, z_{1}, u_{1}\right)$ pour les coordonnées du point dont il s'agit, et en écrivant

$$
p_{1}=y_{1} u_{1}-z_{1}^{2}, \quad q_{1}=z_{1} y_{1}-x_{1} u_{1}, \quad r_{1}=x_{1} z_{1}-y_{1}^{2},
$$

les équations de la droite seront

$$
p_{1} x+q_{1} y+r_{1} z=0, \quad p_{1} y+q_{1} z+r_{1} u=0 .
$$

Or, en considérant en général un€ droite représentée par les équations

$$
\alpha x+\beta y+\gamma z+\delta u=0, \quad \alpha^{\prime} x+\beta^{\prime} y+\gamma^{\prime} z+\delta^{\prime} u=0,
$$

C. V. 
les six quantités

$$
\beta \gamma^{\prime}-\beta^{\prime} \gamma, \quad \gamma \alpha^{\prime}-\gamma^{\prime} \alpha, \quad \alpha \beta^{\prime}-\alpha^{\prime} \beta, \quad \alpha \delta^{\prime}-\alpha^{\prime} \delta, \quad \beta \delta^{\prime}-\beta^{\prime} \delta, \quad \gamma \delta^{\prime}-\gamma^{\prime} \delta,
$$

sont ce que je nomme les coordonnées de la droite (en représentant par $a, b, c, f, g, h$ ces coordonnées, on a l'équation identique $a f+b g+c h=0$, et les coordonnées d'une droite peuvent être des quantités quelconques qui satisfont à cette équation). La condition pour l'involution de six droites est celle-ci, savoir: le déterminant formé avec les coordonnées des six droites est égal à zéro.

Je reviens à la droite qui coupe deux fois la courbe cubique. En écrivant les équations sous la forme

$$
p_{1} x+q_{1} y+r_{1} z+\dot{0} u=0, \quad 0 x+p_{1} y+q_{1} z+r_{1} u=0,
$$

les coordonnées de cette droite seront

$$
p_{1}^{2}, \quad q_{1}^{2}-p_{1} r_{1}, \quad-p_{1} q_{1}, \quad p_{1} r_{1}, \quad q_{1} r_{1}, \quad r_{1}^{2},
$$

savoir, ces coordonnées seront des fonctions linéaires de $\left(p_{1}^{2}, q_{1}^{2}, r_{1}^{2}, q_{1} r_{1}, r_{1} p_{1}, p_{1} q_{1}\right)$. Donc, en considérant six droites dont chacune coupe deux fois la courbe cubique, et en attribuant des significations analogues à $\left(p_{2}, q_{2}, r_{2}\right)$, etc., la condition pour l'involution des six droites se trouve en égalant à zéro le déterminant dont les lignes sont $\left(p_{1}^{2}, q_{1}^{2}, r_{1}^{2}, q_{1} r_{1}, r_{1} p_{1}, p_{1} q_{1}\right),\left(p_{2}^{2}, q_{2}^{2}\right.$, etc.), etc.; condition qui exprime que les six droites

$$
p_{1} x+q_{1} y+r_{1} z=0
$$

dans le plan $u=0$ (ou si l'on veut les six droites $p_{1} y+q_{1} z+r_{1} u=0$ dans le plan $x=0$ ) touchent une même conique. Or la droite

$$
p_{1} x+q_{1} y+r_{1} z=0
$$

est la projection de l'une des six droites sur le plan osculant $u=0$, avec le point $x=y=z=0$ de la courbe cubique comme centre de projection; et si, en prenant un plan osculant quelconque et un point quelconque de la courbe cubique pour plan et centre de projection, nous appelons tout simplement projection une telle projection d'une droite quelconque (le plan osculant et le point de la cubique étant toujours les mêmes), on est conduit au théorème que voici, savoir :

Six droites dont chacune coupe deux fois la même courbe cubique seront en involution, si les projections de ces droites touchent une même conique.

Et de même, pour un nombre quelconque de droites, si les projections touchent une même conique, ces droites seront en involution, c'est-à-dire six quelconques des droites seront des droites en involution.

Il convient de remarquer qu'en considérant six droites quelconques, on peut en général trouver une courbe cubique coupée deux fois par chacune des droites: la condition du théorème est donc, comme cela doit être, une seule relation entre les six droites. Je remarque aussi que cette relation ne dépend nullement du plan osculant ni du point de la courbe cubique choisis pour plan et centre de projection. Réci- 
proquement, en prenant dans un plan osculant quelconque de la courbe cubique un nombre quelconque (six ou plus) de tangentes d'une même conique, et en reprojetant ces tangentes sur la courbe cubique au moyen d'un point quelconque de la courbe comme centre de projection (de manière à obtenir pour reprojection de chaque tangente une droite qui coupe deux fois la courbe cubique), on obtient un système de droites en involution. Le lieu des droites dont chacune coupe deux fois la courbe cubique, et qui sont en involution, est une surface réglée du quatrième ordre qui a la courbe cubique pour courbe double. En effet, si l'équation en coordonnées tangentielles de la conique enveloppée par les droites $p_{1} x+q_{1} y+r_{1} z=0$, etc. (ou, si l'on veut, par les droites $p_{1} y+q_{1} z+r_{1} u=0$, etc.), est

$$
(a, b, c, f, g, h)(p, q, r)^{2}=0,
$$

cette même équation, en y considérant $p, q, r$ comme dénotant $y u-z^{2}, z y-x u, x z-y^{2}$, autrement dit, l'équation,

$$
(a, b, c, f, g, h)\left(y u-z^{2}, z y-x u, x z-y^{2}\right)^{2}=0,
$$

sera celle d'une surface du quatrième ordre ayant la courbe cubique pour courbe double. Et cette surface sera une surface réglée; car en menant par un point quelconque de la surface une droite qui coupe deux fois la courbe cubique, chaque point d'intersection avec la courbe cubique doit compter pour deux points d'intersection avec la surface, et la droite coupe la surface en cinq points, c'est-à-dire que cette droite est située entièrement dans la surface.

J'ai remarqué ailleurs (Camb. and Dubl. Math. Journ., t. vII. (1852), p. 172, [107]) qu'il y a sur une surface réglée de l'ordre $n$ une courbe doub̨le rencontrée par chaque génératrice en $(n-2)$ points. Cette courbe double sera de l'ordre $(n-2)$ au moins, et de l'ordre $\frac{1}{2}(n-1)(n-2)$ au plus; donc, pour $n=4$, la courbe double sera de l'ordre 2 ou 3 , et comme évidemment cette courbe n'est pas une courbe plane, elle sera: ou $1^{\circ}$ deux droites qui ne se rencontrent pas; ou $2^{\circ}$ une courbe cubique en espace. Cette seconde espèce des surfaces réglées du quatrième ordre est celle qui se présente dans la théorie des droites en involution. 